\title{
Targeting protein palmitoylation decreases palmitate-induced sphere formation of human liver cancer cells
}

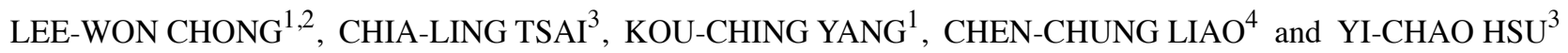 \\ ${ }^{1}$ Division of Hepatology and Gastroenterology, Department of Internal Medicine, \\ Shin Kong Wu Ho Su Memorial Hospital, Taipei; ${ }^{2}$ School of Medicine, Fu Jen Catholic University, \\ New Taipei City; ${ }^{3}$ Institute of Biomedical Sciences, Mackay Medical College, New Taipei City; \\ ${ }^{4}$ Proteomics Research Center, National Yang-Ming University, Taipei, Taiwan, R.O.C.
}

Received October 23, 2019; Accepted April 15, 2020

DOI: $10.3892 / \mathrm{mmr} .2020 .11172$

\begin{abstract}
Although non-alcoholic fatty liver disease (NAFLD) is considered a benign disorder, hepatic steatosis has been proposed to be involved in the tumorigenesis of liver cancer. However, the underlying mechanism for carcinogenesis in fatty liver diseases remains unclear. Cancer stem cells (CSCs) have been hypothesized to serve a key role in tumorigenesis. Tumor formation begins with a subset of heterogeneous cells that share properties with stem cells, such as self-renewal and undifferentiated properties. Our previous study reported that the saturated fatty acid palmitate (PA) significantly enhanced the CSC properties of the HepG2 human liver cancer cell line; however, its underlying mechanisms are unknown. In the present study, a proteomic approach was used to investigate the palmitoylation of proteins in HepG2 CSCs. CSC behavior was induced in HepG2 cells via $200 \mu \mathrm{M}$ PA. Proteomic analysis was performed to identify post-transcriptional modifications of proteins in HepG2 CSCs in response to PA treatment. The present study identified proteins modified by palmitoylation in HepG2 CSC spheres formed following PA treatment. It was therefore hypothesized that palmitoylation may be crucial for CSC sphere formation. Furthermore, the present study demonstrated that two palmitoylation inhibitors, tunicamycin $(5,10$ and $25 \mu \mathrm{g} / \mathrm{ml})$ and 2-bromohexadecanoic acid $(25,50$ and $150 \mu \mathrm{M})$, significantly decreased CSC sphere formation without affecting cell viability. An association was identified between sphere formation capacity and
\end{abstract}

Correspondence to: Dr Yi-Chao Hsu, Institute of Biomedical Sciences, Mackay Medical College, 46 Zhongzheng Road, Sanzhi, New Taipei City, Taiwan, R.O.C.

E-mail: hsuyc@mmc.edu.tw

Dr Chen-Chung Liao, Proteomics Research Center, National Yang-Ming University, 155 Li-Nong Street, Pei-Tou, Taipei, Taiwan, R.O.C.

E-mail: ccliao@ym.edu.tw

Key words: palmitate, HepG2, non-alcoholic steatohepatitis, liver cancer, sphere formation, cancer stem cells tumor-initiating capacity of CSCs. The results of the present study demonstrated that protein palmitoylation may influence the PA-induced CSC tumor-initiating capacity, and that the inhibition of palmitoylation may be a suitable chemopreventive strategy for treating patients with NAFLD.

\section{Introduction}

Non-alcoholic fatty liver disease (NAFLD) is a metabolic disease that is a global public health concern. From 1989 to 2015 , the incidence rate of NAFLD was 28.01-52.34 per 1,000 people for Asia and Israel (1). In 2018, the prevalence of NAFLD was $\sim 25 \%$ of the world population (2). The clinical process of NAFLD includes simple steatosis, steatohepatitis, hepatic fibrosis/cirrhosis and hepatocellular carcinoma (HCC) (3). The two-hit hypothesis of non-alcoholic steatohepatitis (NASH) suggests that hepatic steatosis ('first hit') is a prerequisite for subsequent events ('second hits') that lead to hepatic injury (4). Free fatty acids (FFAs) are the primary mediators of excessive lipid accumulation in the liver. Notably, circulating FFA levels are significantly increased in patients with NAFLD, and evidence indicates that plasma FFAs are associated with disease severity (5). Excess FFA-induced hepatic injury results from the limited capacity of hepatocytes to transform FFA into triglycerides in lipid droplets (5). Excess lipid droplets in cells can result in mitochondrial dysfunction and oxidative stress, potentially contributing to hepatic inflammation and fibrosis in NASH (5).

The initiation of cancer may be the result of mutations in stem cells that interfere with differentiation. Certain tumor cells share properties with stem cells, which indicates that cancer stem cells (CSCs) may be responsible for cancer initiation and progression. These distinctive properties of CSCs are the capacities for self-renewal and cell proliferation (spherogenesis), which are primary causes of cancer recurrence and metastasis (6). The sonic hedgehog (Shh) signaling pathway and stemness-connected transcription factors (such as Sox 2 and Oct4) are primarily responsible for CSC proliferation $(7,8)$. CSCs have been hypothesized to be responsible for the carcinogenesis of HCC. An increasing number of studies have demonstrated that growth factors, such as epidermal growth factor (EGF) or fibroblast growth factor 2 (FGF2), can stimulate cell proliferation 
of CSCs and sphere formation in a three-dimensional culture system. Sphere formation is a practical approach for enriching certain CSC subpopulations with self-renewal properties and CSC marker expression levels $(9,10)$.

Research has demonstrated that liver CSC subpopulations can be isolated by certain cell surface markers, specifically CD133 (11,12), CD90 (13-15), CD44 (16), the epithelial cell adhesion molecule (17) and CD13 (18). A previous study demonstrated that undifferentiated multipotent neural stem cells could be expanded via suspension using sphere assay (19). Sphere assays have been used to study adult stem cells in numerous organs and tissues, including the liver, nerves, prostate and mammary (9,20-22). Furthermore, sphere formation assay is widely recognized for its ability to enrich potential CSC subpopulations via stimulation of EGF or FGF2 (9,23-25).

Our previous study reported that exposure of hepatocytes to palmitate (PA) induced reactive oxygen species (ROS) production, $\mathrm{NF \kappa B}$ activation and inflammatory cytokine expression levels in primary rat hepatocytes (PRHs) (26) and HepG2 cells (27). This increase in proinflammatory cytokines induced by PA could serve a key role in hepatic stellate cell activation and the development of hepatic fibrosis. Furthermore, it was demonstrated that PA induced CSC sphere formation in HepG2 cells but not in PRHs. Conversely, inhibition of NFאB activity significantly limited HepG2 CSC sphere formation (27). In the present study, it was hypothesized that PA would induce CSC properties via palmitoylation, as palmitoylation has been demonstrated to regulate CSC markers, estrogen receptors and epidermal growth factor (EGF) receptors (28). Liquid chromatography-tandem mass spectrometry (LC/MS/MS) was used to identify the palmitoylation of proteins in PA-treated HepG2 CSC spheres. The present study demonstrated that numerous proteins were palmitoylated in CSC spheres formed following PA treatment. After treating the HepG2 cells with two palmitoylation inhibitors, tunicamycin and 2-bromohexadecanoic acid, the PA-treated CSC spheres were notably inhibited. Taken together, the results of the present study verified the hypothesis that PA-induced palmitoylation is key for HepG2 CSC sphere formation. Thus, the inhibition of palmitoylation may be a chemopreventive strategy for use in treating patients with NAFLD.

\section{Materials and methods}

Chemicals. PA (cat. no. 102553) and BSA were acquired from Sigma-Aldrich (Merck KGaA). PA stock solution was prepared as previously described (27). In brief, $400 \mathrm{mM}$ PA stock solution was prepared in DMSO. The 3\% (w/v) FFA-free BSA solution was prepared in DMEM and maintained at $55^{\circ} \mathrm{C}$. A $5 \mathrm{mM}$ FFA/BSA solution was prepared by dissolving the $128.2 \mathrm{mg}$ of PA in $1 \mathrm{ml}(500 \mathrm{mM})$ ethanol and heating at $70^{\circ} \mathrm{C}$ for $10 \mathrm{~min}$. $10 \% \mathrm{BSA}$ solution was made in DMEM medium, filtered and incubated at $37^{\circ} \mathrm{C}$ for $10 \mathrm{~min}$. Subsequently, $10 \mathrm{ul}$ of dissolved PA was added to $990 \mu \mathrm{l}$ of $10 \% \mathrm{BSA}$, and the solution was vortexed and incubated at $55^{\circ} \mathrm{C}$ for $30 \mathrm{~min}$. The final PA concentration in BSA solution was $5 \mathrm{mM}$. The FFA/BSA solution was diluted to the concentration used in the experiments using DMEM. All solutions were freshly prepared before use. The $1 \mathrm{mg} / \mathrm{ml}$ tunicamycin and $1 \mathrm{mM}$ 2-bromohexadecanoic acid (cat. nos. T7765 and 21604, respectively; both
Sigma-Aldrich; Merck KGaA) stock solutions were prepared in ethanol and stored at $-20^{\circ} \mathrm{C}$.

Cell culture. The human liver cancer cell line HepG2 was obtained from the Bioresource Collection and Research Center. Authentication of the HepG2 cell line used in the present study was performed via the short tandem repeat-PCR method. Cells were maintained as a monolayer in culture medium (Thermo Fisher Scientific, Inc.), supplemented with $10 \%$ FBS (Thermo Fisher Scientific, Inc.), $100 \mathrm{IU} / \mathrm{ml}$ penicillin and $100 \mu \mathrm{g} / \mathrm{ml}$ streptomycin. Cells were maintained at $37^{\circ} \mathrm{C}$ in a humidified $5 \% \mathrm{CO}_{2}$ incubator. During sphere assays, cells were harvested and washed with PBS to remove serum. Cells were then suspended in ultralow-attachment 6-well plates (Corning, Inc.) at a density of 5,000 cells/well. Serum-free DMEM/F12 culture medium was supplemented with $100 \mathrm{IU} / \mathrm{ml}$ penicillin, $100 \mu \mathrm{g} / \mathrm{ml}$ streptomycin, $20 \mathrm{ng} / \mathrm{ml}$ human recombinant EGF, $10 \mathrm{ng} / \mathrm{ml}$ human recombinant FGF2, 2\% B27 supplement and $1 \% \mathrm{~N}_{2}$ supplement (Thermo Fisher Scientific, Inc.). Mycoplasma testing was performed for the cell lines used. The maintenance and culture of HepG2 cells were performed as previously described (27). Cell morphology was observed and recorded using a light microscope (magnification, x40) and the most representative image was selected.

Oil Red $O$ staining. After HepG2 cells were exposed to $200 \mu \mathrm{M}$ PA (Sigma-Aldrich; Merck KGaA) for $12 \mathrm{~h}$ at $37^{\circ} \mathrm{C}$, cells were stained with Oil Red $\mathrm{O}$ to investigate the amount of fat accumulation in cells. Briefly, cells were washed with PBS and fixed in $10 \%$ neutral formalin at room temperature (RT) for $30 \mathrm{~min}$. After two washes with propylene glycol, 10\% Oil Red $\mathrm{O}$ was added and cells were stained for $7 \mathrm{~min}$ at $\mathrm{RT}$ before being washed with $85 \%$ propylene glycol. Cells were rinsed in distilled water and counterstained with $10 \%$ hematoxylin solution at RT for $8 \mathrm{~min}$. A total of three images were captured per well using a light microscope (magnification, $x 40$ ) and the most representative image was selected.

CSC sphere formation assay. After cells were cultured under serum-free medium supplemented with EGF and FGF2 for sphere formation, spheres were collected via centrifugation at $700 \mathrm{x} \mathrm{g}$ at RT for $3 \mathrm{~min}$. Subsequently, cells were dissociated using trypsin-EDTA and mechanically disrupted with a pipette to obtain single cells. The sphere-derived single cells were then centrifuged at $700 \mathrm{xg}$ at RT for $3 \mathrm{~min}$ to remove the trypsin-EDTA and resuspended in serum-free medium for cell counting using trypan-blue exclusion assay (Thermo Fisher Scientific, Inc.) using a light microscope (magnification, $\mathrm{x} 100)$ and the replication of sphere assays. The spheres were passaged every 7 days until they reached a diameter of $100 \mu \mathrm{m}$.

Mass spectrometry. PA-treated HepG2 CSCs were collected to extract protein lysates using lysis buffer [1 $\mathrm{ml}$ ice-cold buffer (20 mM Tris-Hcl, pH 7.4; $150 \mathrm{mM} \mathrm{NaCl} ; 1 \%$ (v/v) Triton X-100; $0.5 \%$ (v/v) Nonidet P40; $1 \mathrm{mM}$ EDTA; $1 \mathrm{mM}$ PMSF; $2 \mu \mathrm{g} / \mathrm{ml}$ Antipain C; $50 \mu \mathrm{g} / \mathrm{ml}$ tosyl phenylalanyl chloromethyl ketone; $10 \mu \mathrm{g} / \mathrm{ml}$ Leupeptin; $1 \mathrm{mM} \mathrm{NaF} ; 1 \mathrm{mM}$ $\mathrm{NaVO}_{3} ; 5 \mathrm{mM} \mathrm{Na} \mathrm{P}_{2} \mathrm{O}_{7}$ )]. A total of $10 \mu \mathrm{g}$ of protein lysates were further separated using 10\% SDS-PAGE and stained with $0.25 \%$ Coomassie brilliant blue R-250 solution (Bio-Rad 
A

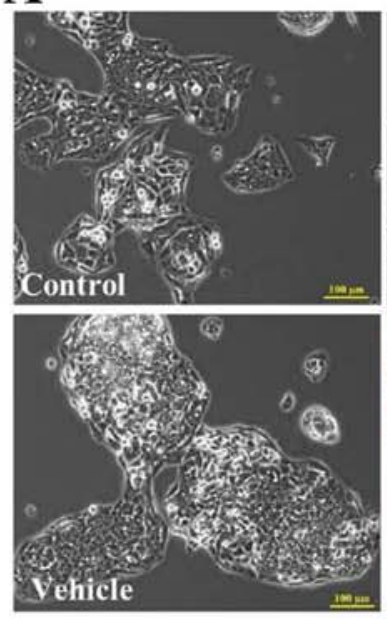

B
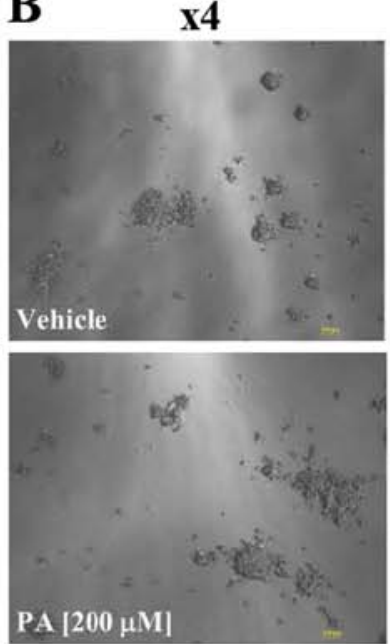

$\mathrm{C}$

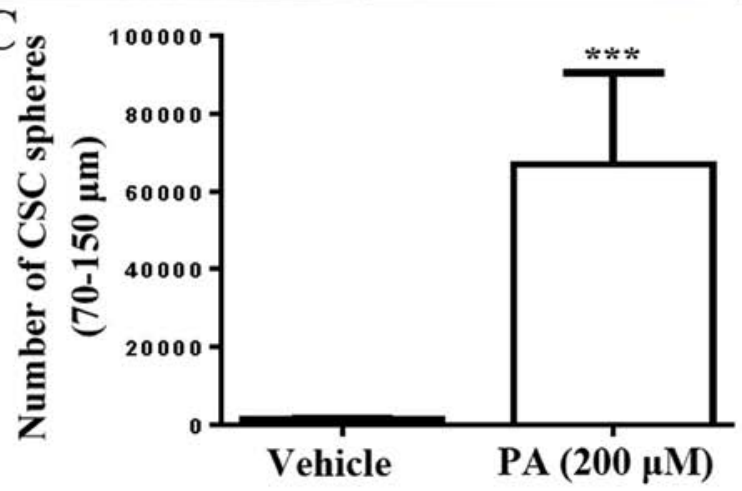

Figure 1. Effects of PA on human HepG2 cells. (A) PA did not affect cel growth but induced lipid accumulation in HepG2 cells under 10\% FBS culture medium. Scale bar, $100 \mu \mathrm{m}$. The 3\% (w/v) FFA-free BSA solution was prepared in DMEM (vehicle) and was used to prepare PA solution (B) Effects of PA on human HepG2 CSCs under sphere formation culture conditions. Scale bar, $100 \mu \mathrm{m}$. (C) PA significantly increased the number of human HepG2 CSC spheres. Data are presented as the mean \pm standard error of the mean; $\mathrm{n}=3$. ${ }^{* * *} \mathrm{P}<0.001$. $\mathrm{PA}$, palmitate; $\mathrm{CSC}$, cancer stem cell.

Laboratories, Inc.) at RT for $1 \mathrm{~h}$. All subsequent procedures for in-gel digestion and mass spectrometry were performed as previously described (29).

Statistical analysis. Data are presented as the mean \pm standard error of the mean. One-way analysis of variance was performed
A

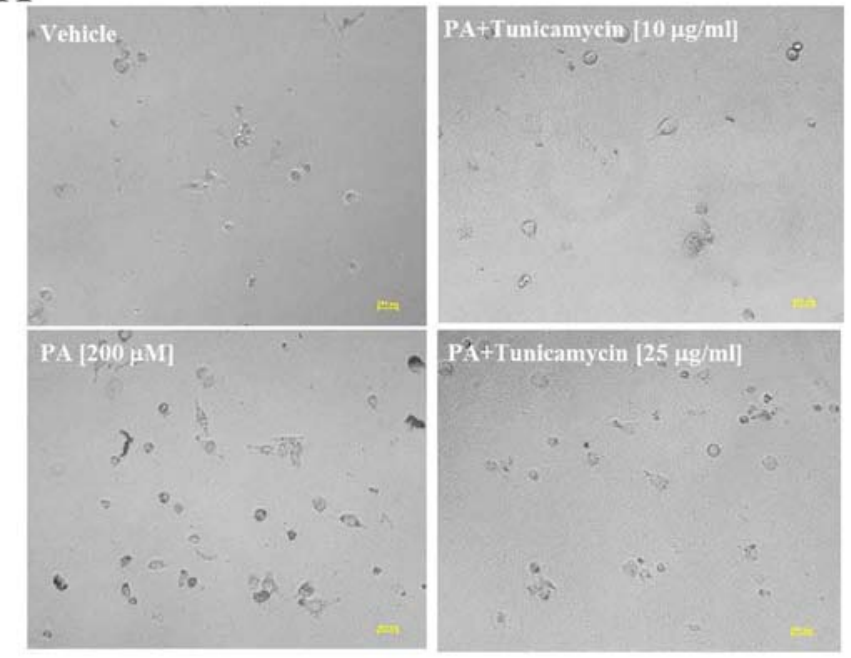

B

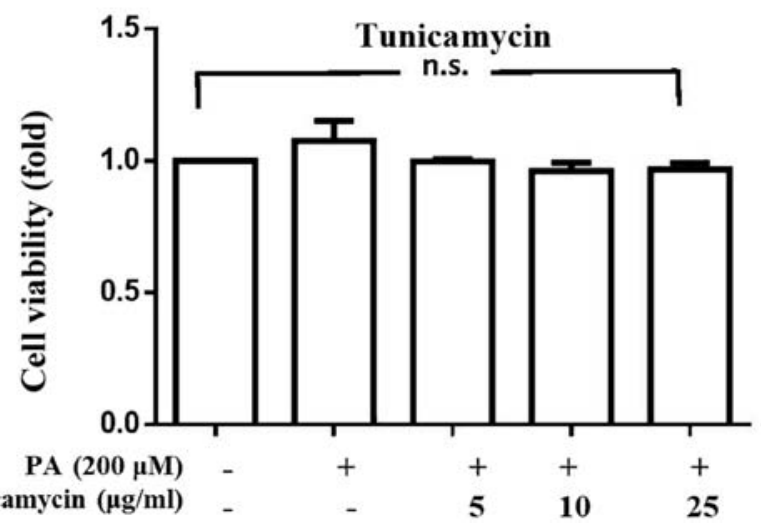

Figure 2. Effects of tunicamycin on viability of human HepG2 cells in $10 \%$ FBS culture medium. (A) Morphology of tunicamycin-treated HepG2 cells. Scale bar, $100 \mu \mathrm{m}$. Tunicamycin did not alter the morphology of HepG2 under $10 \%$ serum culture condition. (B) Tunicamycin $(5,10$ and $25 \mu \mathrm{g} / \mathrm{ml}$ ) did not affect viability of PA-treated human HepG2 cells in $10 \%$ FBS culture medium. Data are presented as the mean \pm standard error of the mean; $n=3$. PA, palmitate; n.s., not significant.

to compare the statistical differences in biochemical and molecular parameters. The post hoc test performed was Tukey's multiple comparisons test. PRISM software (version 6.0; GraphPad Software, Inc.) was used for this purpose. A total of three independent experiments were performed. $\mathrm{P}<0.05$ was considered to indicate a statistically significant difference.

\section{Results}

Effects of PA on HepG2 cells. The present study investigated the underlying mechanisms of the effects of PA on human HepG 2 cells. The results indicated that, at a concentration of $200 \mu \mathrm{M}$, PA did not affect the growth and survival rate of HepG2 cells but did induce morphological changes in HepG2 cells in the $10 \%$ FBS culture medium (Fig. 1A). The results of Oil Red $\mathrm{O}$ staining indicated that lipid droplets accumulated inside HepG2 cells following PA treatment (Fig. 1A). Furthermore, the present study investigated the effects of PA on the cancer sphere formation ability of HepG2 cells; it was demonstrated that PA treatment significantly increased the cancer sphere formation ability of HepG2 cells $(n=3 ; P<0.001)$. Representative images are presented in Fig. $1 \mathrm{~B}$ and $\mathrm{C}$. 


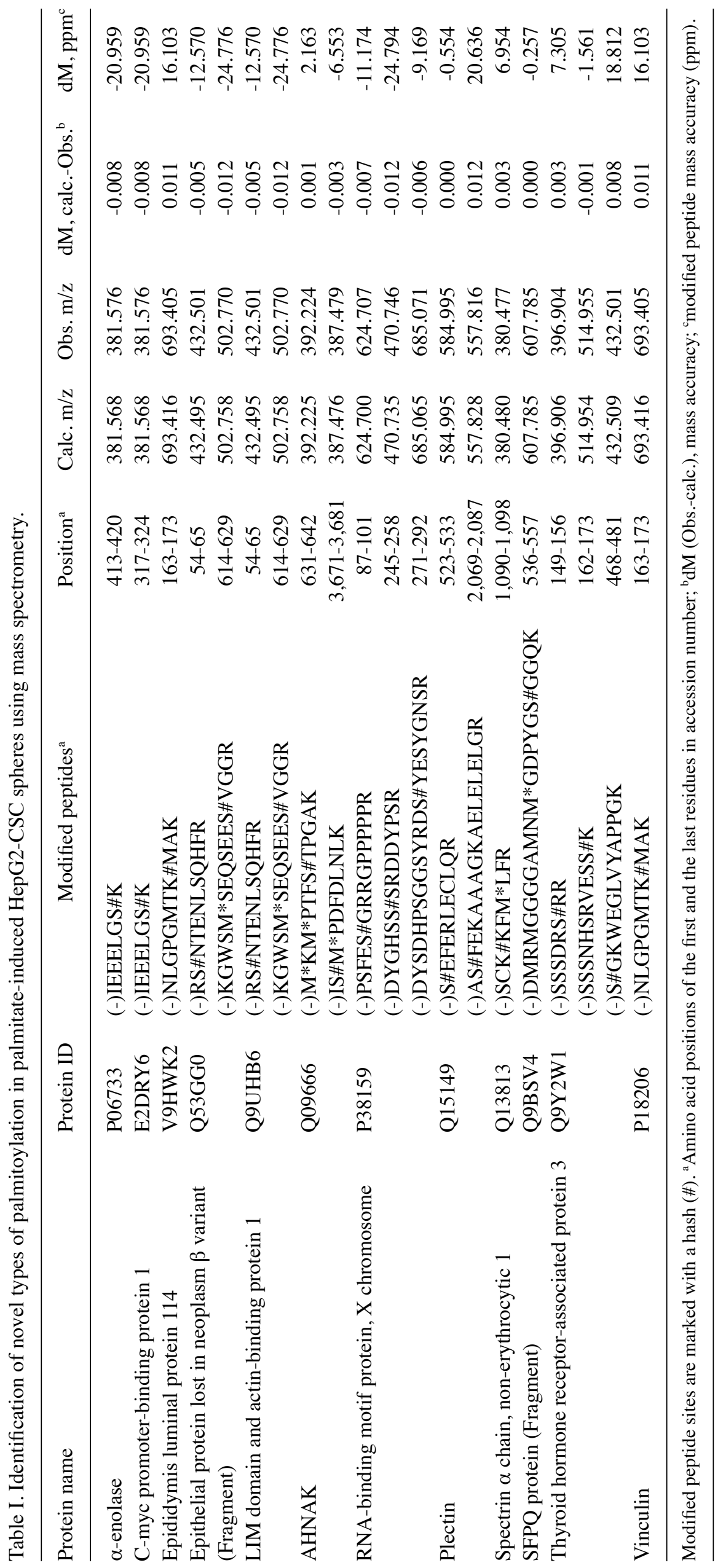


$\mathbf{A}$

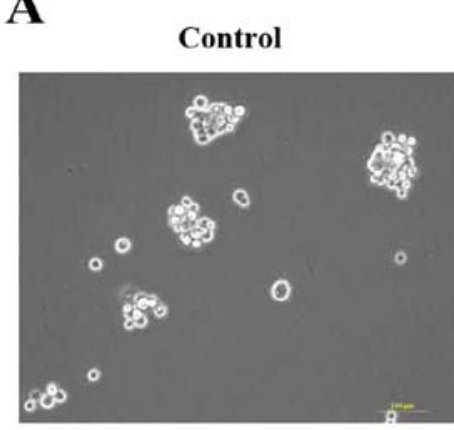

PA $(200 \mu M)$
PA $(200 \mu \mathrm{M})+$

tunicamycin $(5 \mu \mathrm{M})$

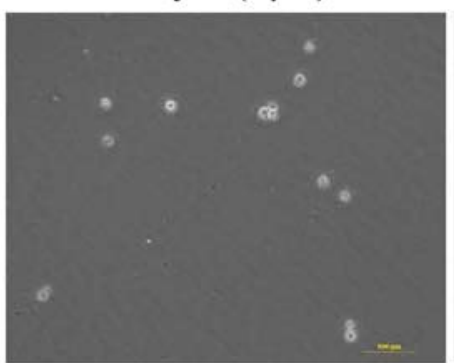

PA $(200 \mu \mathrm{M})+$

tunicamycin $(10 \mu \mathrm{M})$

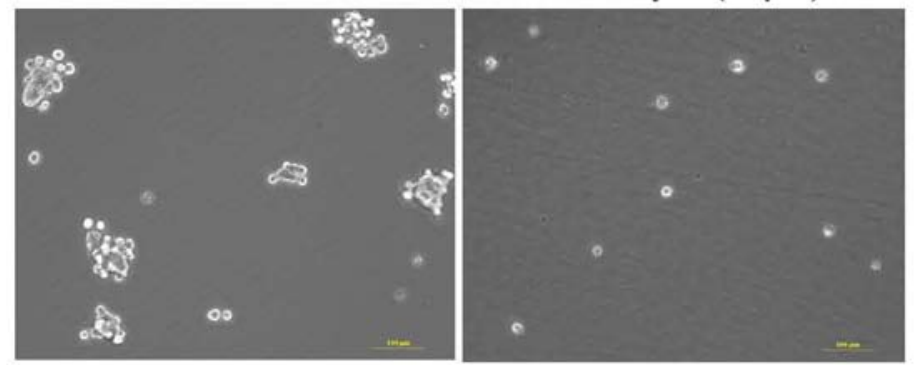

B
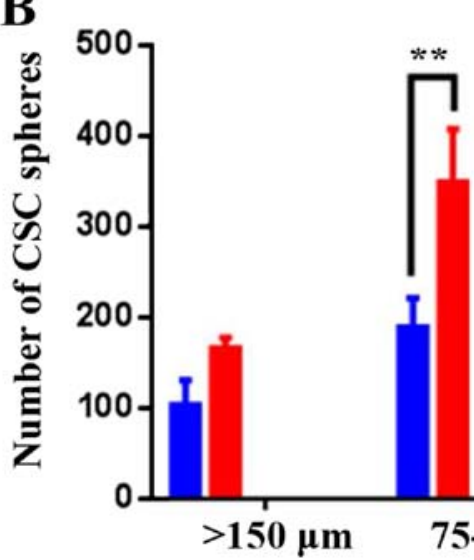

Vehicle

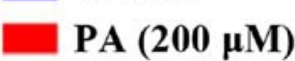

PA tunicamycin $(5 \mu \mathrm{M})$

PA tunicamycin $(10 \mu \mathrm{M})$

PA tunicamycin $(25 \mu \mathrm{M})$

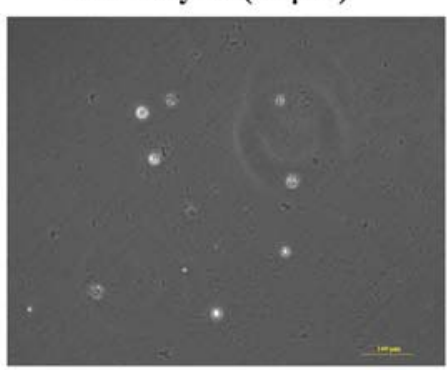

PA $(200 \mu \mathrm{M})+$

tunicamycin $(25 \mu \mathrm{M})$

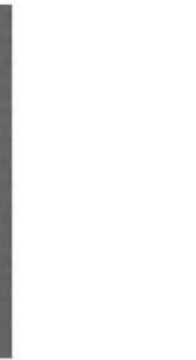


A

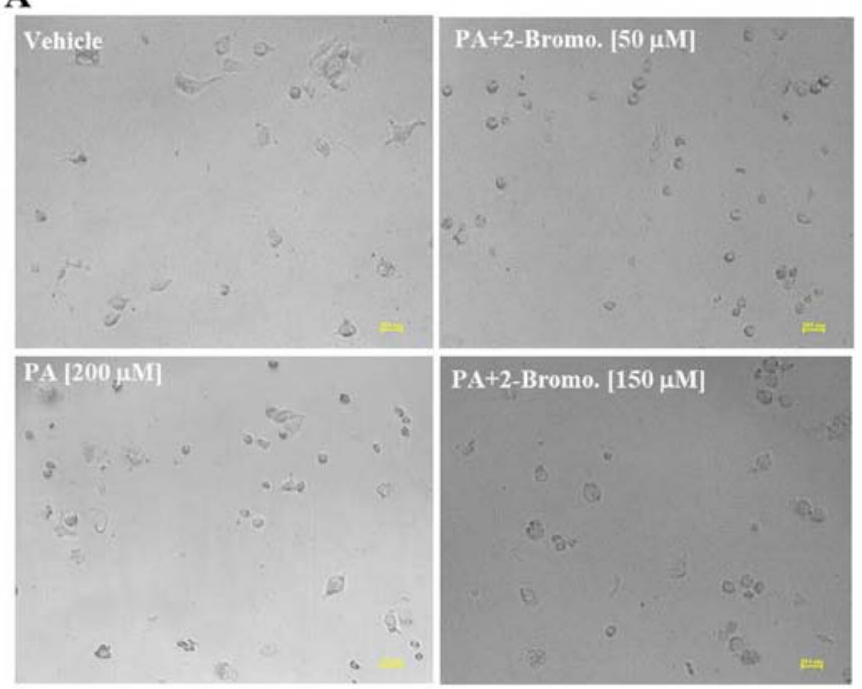

B

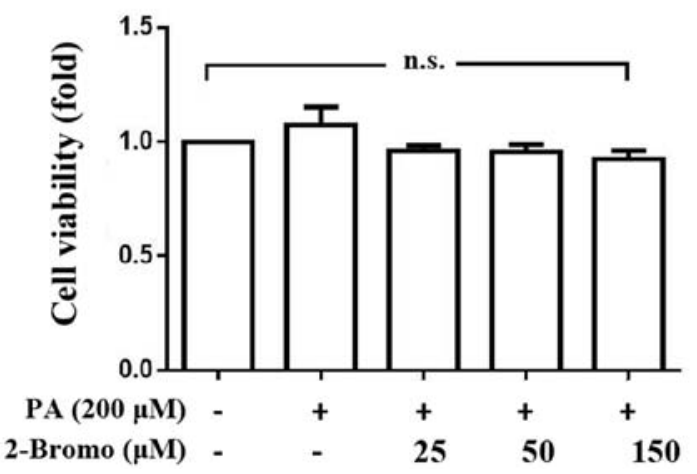

Figure 4. Effects of 2-bromo on viability of human HepG2 cells in $10 \%$ FBS culture medium. (A) Cell morphology of 2-bromo-treated HepG2 cells Scale bar, $100 \mu \mathrm{m}$. (B) 2-Bromo $(25,50$ and $150 \mu \mathrm{M})$ did not affect viability of PA-treated human HepG2 cells in the 10\% FBS culture medium. Data are presented as the mean \pm standard error of the mean; $n=3$. 2-Bromo, 2-bromodecahexanoic acid; PA, palmitate; n.s., not significant.

PA-induced HepG2-CSC sphere formation (75-150 $\mu \mathrm{m})$ at these concentrations (Fig. 5).

\section{Discussion}

NAFLD is the most common type of liver disease worldwide and is expected to become a primary cause of HCC in the near future. Previous research has investigated the effects and underlying mechanisms of saturated FFAs, such as PA, on liver cells. Our previous study demonstrated that PA increased intracellular hydrogen peroxide levels in PRHs and the human liver cancer cell line HepG2, and induced the expression levels of proinflammatory cytokines, such as TNF- $\alpha$, IL6 and intercellular adhesion molecule 1. Furthermore, PA activated hepatic stellate cells and contributed to steatosis-associated hepatic fibrogenesis (30). In addition, PA has been reported to significantly increase CSC sphere formation in HepG2 cells but not in PRH (27). Furthermore, PA (50, 100 and $200 \mu \mathrm{M})$ activated the expression levels of pluripotent genes, including Sox 2, Oct 4 and Shh in a dose-dependent manner, and $200 \mu \mathrm{M}$ PA significantly increased the sphere formation capacity of HepG2 cells (27). In the present study, $200 \mu \mathrm{M}$ PA significantly increased the sphere formation capacity of HepG2 cells.
Notably, in a previous study, PA $(10,50,100$ and $200 \mu \mathrm{M})$ dose-dependently induced production of ROS in primary hepatocytes and HepG2 cells but did not affect the viability of primary hepatocytes and HepG2 cells (30). Furthermore, PA not only induced proinflammatory responses in hepatocytes and HepG2 cells (30) but also activated the CSC-like properties of HepG2 cells (27) to increase sphere formation. However, long-term PA treatment may cause cells to become senescent, as certain types of cell are prone to senescence rather than apoptosis following high exogenous stress (31).

The primary saturated fatty acid in HepG2 cells is PA, which undergoes post-translation palmitoylation. Research indicates that palmitoylation modulates protein function at all stages of protein processing (26). In addition, other saturated fatty acids, such as myristic and stearic acid, and unsaturated fatty acids, such as oleic and arachidonic acid, also undergo post-translational modification. The present study used LC/MS/MS to identify palmitoylation-modified proteins in PA-treated HepG2 CSC spheres (Fig. 1); numerous proteins were demonstrated to be palmitoylated.

Tunicamycin is a nucleoside antibiotic that can inhibit protein palmitoylation. Tunicamycin has been reported to inhibit palmitoylation of the endothelial isoform of nitric oxide synthase, promote nitric oxide synthesis in aortic endothelial cells and increase the influx of $\mathrm{Ca}^{2+}$ across the plasma membrane (32). In the present study, tunicamycin did not affect HepG2 cell viability in 10\% FBS culture medium but decreased PA-induced formation of HepG2 CSC spheres in the sphere formation culture medium. Although 2-bromohexadecanoic acid (2-bromopalmitate) is used as a non-selective inhibitor of lipid metabolism, it has been demonstrated to be a general inhibitor of protein S-palmitoylation. It has been hypothesized that 2-bromopalmitate is converted to 2-bromopalmitoyl-CoA in cells. Notably, both 2-bromopalmitate and 2-bromopalmitoyl-CoA interact with DHHC palmitoyl acyl transferases, which catalyze protein S-palmitoylation. The present study demonstrated that 2-bromohexadecanoic acid did not affect HepG2 cell viability in the 10\% FBS culture medium, but decreased PA-induced formation of HepG2 CSC spheres in the sphere formation culture medium. Neither 2-bromopalmitate nor tunicamycin affected viability of HepG2 cells under serum-containing culture conditions. However, they both significantly decreased the number of HepG2 spheres formed under serum-free culture conditions. One explanation for this finding is that serum supplementation may activate cell survival to eliminate ER stress when cells are treated with 2-bromopalmitate or tunicamycin. The present study investigated the effects of PA and two inhibitors, 2-bromopalmitate and tunicamycin, on palmitoylation. Although 2-bromopalmitate is traditionally used as an inhibitor of PA, its effects extend beyond protein palmitoylation. Tunicamycin can also function as an inhibitor of protein $\mathrm{N}$-glycosylation and induce cellular ER stress. Further research is necessary to determine other mechanisms of 2-bromopalmitate and tunicamycin and to investigate their ability to decrease PA-induced protein palmitoylation.

The effects of tunicamycin at concentrations of 5, 10 and $25 \mu \mathrm{g} / \mathrm{ml}$, and 2-bromopalmitate at a concentration of $150 \mu \mathrm{M}$ observed in the present study are consistent with those reported in the literature $(6,7)$. Sobocińska et al (33) used 2-bromopalmitate (125 and $250 \mu \mathrm{M}$ ) to inhibit lipopolysaccharide-induced 
A
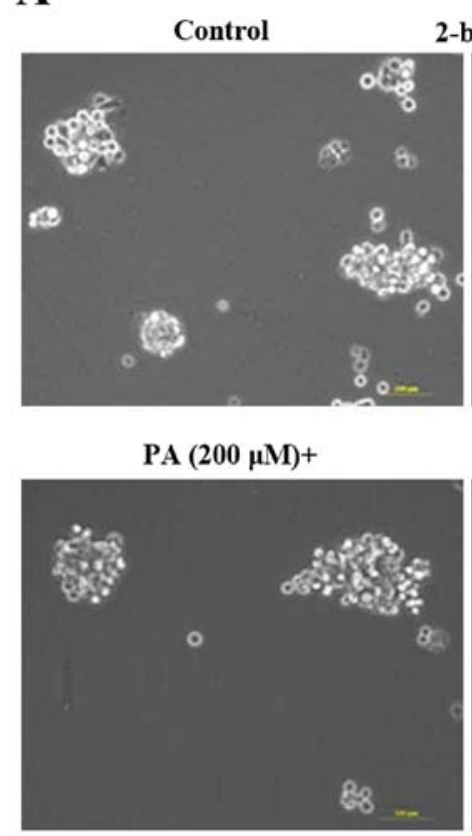

B
PA $(200 \mu \mathrm{M})+$

-bromohexadecanoic acid $(25 \mu \mathrm{M})$

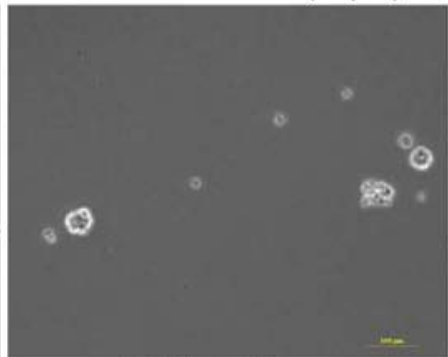

$\mathrm{PA}(200 \mu \mathrm{M})+$

2-bromohexadecanoic acid $(50 \mu \mathrm{M})$

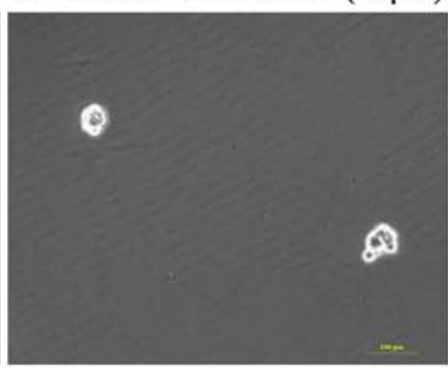

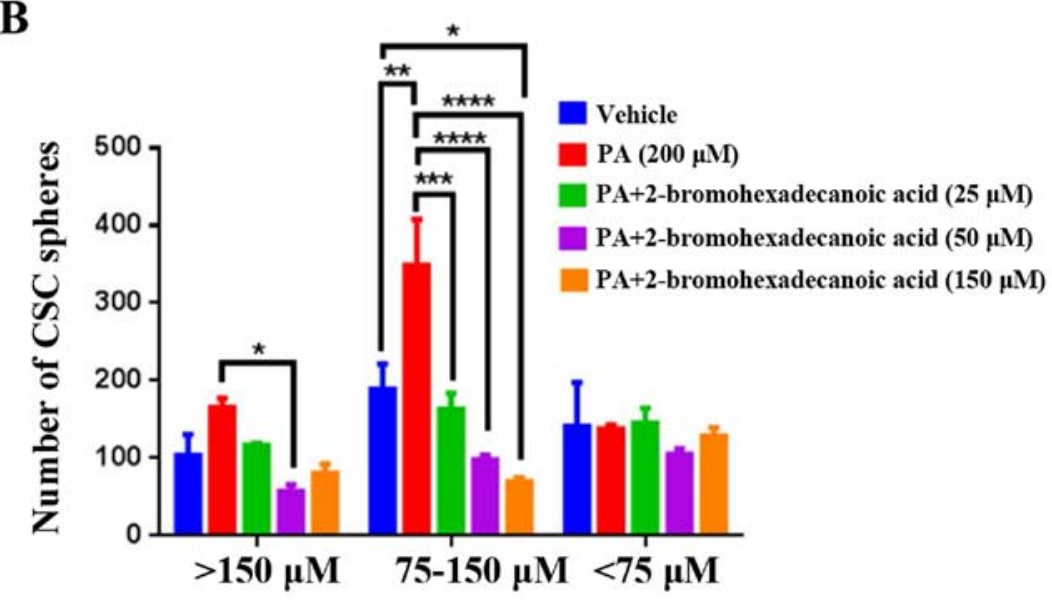

Figure 5. Effects of 2-bromohexadecanoic acid on CSC sphere formation of human HepG2 cells under CSC sphere formation culture condition. (A) Sphere morphology of tunicamycin-treated HepG2 cells. Scale bar, $100 \mu \mathrm{m}$. (B) 2-Bromohexadecanoic acid (25,50 and $150 \mu \mathrm{M})$ significantly decreased the number of HepG2 CSC spheres (75-150 $\mu \mathrm{m}$ and $>150 \mu \mathrm{m}$ ). Data are presented as the mean \pm standard error of the mean; $\mathrm{n}=3 .{ }^{*} \mathrm{P}<0.05,{ }^{* *} \mathrm{P}<0.01,{ }^{* * * *} \mathrm{P}<0.001,{ }^{* * * * *} \mathrm{P}<0.0001$. CSC, cancer stem cell.

S-palmitoylation and activation of PI4KII $\beta$; this activation generated phosphatidylinositol-4-phosphate, which is involved in signaling pathways controlling the production of proinflammatory cytokines. Patterson and Skene (34) demonstrated that tunicamycin $(10 \mu \mathrm{g} / \mathrm{ml})$ significantly inhibited growth cone protein palmitoylation in intact neuronal cells. On the other hand, the results of the present study demonstrated that PA may induce protein palmitoylation and CSC properties via inducing expression levels of carnitine O-palmitoyltransferase 1 and the CSC marker CD44. Additionally, tunicamycin and 2-bromopalmitate treatment notably suppressed the expression levels of carnitine O-palmitoyltransferase 1 and CD44, respectively (Data S1), indicating a novel mechanism of tunicamycin and 2-bromopalmitate in the inhibition of palmitoylation.

In breast cancer, palmitoylation has been hypothesized to control the function of commonly dysregulated genes, including estrogen and EGF receptors, and CSC marker proteins. Palmitoylation also regulates the formation of complexes, such as integrins, at the plasma membrane (26). However, it is unclear whether aberrant palmitoylation can contribute to tumor initiation and growth in the liver. The results of the present study indicated that PA-mediated palmitoylation may be a key factor for inducing CSC activation in the fatty liver of patients with NAFLD. In conclusion, the development of specific inhibitors for the suppression of PA-mediated palmitoylation may present a promising chemopreventive strategy to treat patients with NAFLD.

\section{Acknowledgements}

Not applicable.

\section{Funding}

The present study was funded by the Shin Kong Wu Ho Su Memorial Hospital (grant no. SKH 8302-105-DR-06), 
the Ministry of Science and Technology of Taiwan Government (grant nos. 107-2314-B-715-004-MY3, 103-2314-B-715-001-MY2, 104-2314-B-715-003-MY3, 105-2320-B-039-059-MY3 and 108-2320-B-039-013) and Mackay Medical College (grant nos. 1052B07, 1051B23, 1061B09, 1071B12 and 1081E03).

\section{Availability of data and materials}

The datasets used and/or analyzed during the current study are available from the corresponding author on reasonable request.

\section{Authors' contributions}

LWC, YCH and CCL were involved in the conceptualization, supervision and funding acquisition of the present study. LWC, $\mathrm{YCH}$ was involved in project administration. LWC, CLT, KCY, CCL and YCH were involved in the investigation, methodology and analysis of the present study, and in the writing of the manuscript. The final manuscript was read and approved by LWC, CLT, KCY, CCL and YCH.

\section{Ethics approval and consent to participate}

Not applicable.

\section{Patient consent for publication}

Not applicable.

\section{Competing interests}

The authors declare that they have no competing interests.

\section{References}

1. Younossi ZM, Koenig AB, Abdelatif D, Fazel Y, Henry L and Wymer M: Global epidemiology of nonalcoholic fatty liver disease-Meta-analytic assessment of prevalence, incidence, and outcomes. Hepatology 64: 73-84, 2016.

2. Younossi Z, Tacke F, Arrese M, Chander Sharma B, Mostafa I, Bugianesi E, Wai-Sun Wong V, Yilmaz Y, George J, Fan J and Vos MB: Global perspectives on non-alcoholic fatty liver disease and non-alcoholic steatohepatitis. Hepatology 69: 2672-2682, 2019.

3. Angulo P and Lindor KD: Treatment of non-alcoholic steatohepatitis. Best Pract Res Clin Gastroenterol 16: 797-810, 2002.

4. Malaguarnera M, Di Rosa M, Nicoletti F and Malaguarnera L: Molecular mechanisms involved in NAFLD progression. J Mol Med (Berl) 87: 679-695, 2009.

5. Leamy AK, Egnatchik RA and Young JD: Molecular mechanisms and the role of saturated fatty acids in the progression of non-alcoholic fatty liver disease. Prog Lipid Res 52: 165-174, 2013.

6. Cao L, Zhou Y, Zhai B, Liao J, Xu W, Zhang R, Li J, Zhang Y, Chen L, Qian H, et al: Sphere-forming cell subpopulations with cancer stem cell properties in human hepatoma cell lines. BMC Gastroenterol 11: 71, 2011.

7. Huang P, Qiu J, Li B, Hong J, Lu C, Wang L, Wang J, Hu Y, Jia W and Yuan Y: Role of Sox 2 and Oct4 in predicting survival of hepatocellular carcinoma patients after hepatectomy. Clin Biochem 44: 582-589, 2011.

8. Sun C, Sun L, Li Y, Kang X, Zhang S and Liu Y: Sox 2 expression predicts poor survival of hepatocellular carcinoma patients and it promotes liver cancer cell invasion by activating Slug. Med Oncol 30: 503, 2013.

9. Yin X, Li YW, Zhang BH, Ren ZG, Qiu SJ, Yi Y and Fan J: Coexpression of stemness factors Oct4 and Nanog predict liver resection. Ann Surg Oncol 19: 2877-2887, 2012.
10. Ma XL, Sun YF, Wang BL, Shen MN, Zhou Y, Chen JW, Hu B, Gong ZJ, Zhang X, Cao Y, et al: Sphere-forming culture enriches liver cancer stem cells and reveals Stearoyl-CoA desaturase 1 as a potential therapeutic target. BMC Cancer 19: 760, 2019

11. Stafman LL, Williams AP, Garner EF, Aye JM, Stewart JE, Yoon KJ, Whelan K and Beierle EA: Targeting PIM kinases affects maintenance of CD133 tumor cell population in hepatoblastoma. Transl Oncol 12: 200-208, 2019.

12. Maehara O, Ohnishi S, Asano A, Suda G, Natsuizaka M, Nakagawa K, Kobayashi M, Sakamoto N and Takeda H: Metformin regulates the expression of CD133 through the AMPK-CEBP $\beta$ pathway in hepatocellular carcinoma cell lines. Neoplasia 21: 545-556, 2019.

13. Chen WC, Chang YS, Hsu HP, Yen MC, Huang HL, Cho CY, Wang CY, Weng TY, Lai PT, Chen CS, et al: Therapeutics targeting CD90-integrin-AMPK-CD133 signal axis in liver cancer. Oncotarget 6: 42923-42937, 2015.

14. He J, Liu Y, Zhu T, Zhu J, Dimeco F, Vescovi AL, Heth JA, Muraszko KM, Fan X and Lubman DM: CD90 is identified as a candidate marker for cancer stem cells in primary high-grade gliomas using tissue microarrays. Mol Cell Proteomics 11: M111.010744, 2012.

15. Zhang K, Che S, Pan C, Su Z, Zheng S, Yang S, Zhang H, Li W Wang W and Liu J: The SHH/Gli axis regulates CD90-mediated liver cancer stem cell function by activating the IL6/JAK2 pathway. J Cell Mol Med 22: 3679-3690, 2018.

16. Wei S, Liu K, He Q, Gao Y and Shen L: PES1 is regulated by CD44 in liver cancer stem cells via miR-105-5p. FEBS Lett 593: 1777-1786, 2019.

17. Yamashita T, Honda M, Nakamoto Y, Baba M, Nio K, Hara Y, Zeng SS, Hayashi T, Kondo M, Takatori H, et al: Discrete nature of EpCAM+ and CD90+ cancer stem cells in human hepatocellular carcinoma. Hepatology 57: 1484-1497, 2013.

18. Christ B, Stock P and Dollinger MM: CD13: Waving the flag for a novel cancer stem cell target. Hepatology 53: 1388-1390, 2011.

19. Reynolds BA and Weiss S: Clonal and population analyses demonstrate that an EGF-responsive mammalian embryonic CNS precursor is a stem cell. Dev Biol 175: 1-13, 1996.

20. Dontu G, Abdallah WM, Foley JM, Jackson KW, Clarke MF, Kawamura MJ and Wicha MS: In vitro propagation and transcriptional profiling of human mammary stem/progenitor cells. Genes Dev 17: 1253-1270, 2003.

21. Suzuki A, Oyama K, Fukao K, Nakauchi H and Taniguchi H: Establishment of clonal colony-forming assay system for pancreatic stem/progenitor cells. Cell Transplant 11: 451-453, 2002.

22. Shi X, Gipp J and Bushman W: Anchorage-independent culture maintains prostate stem cells. Dev Biol 312: 396-406, 2007.

23. Ponti D, Costa A, Zaffaroni N, Pratesi G, Petrangolini G, Coradini D, Pilotti S, Pierotti MA and Daidone MG: Isolation and in vitro propagation of tumorigenic breast cancer cells with stem/progenitor cell properties. Cancer Res 65: 5506-5511, 2005.

24. Gou S, Liu T, Wang C, Yin T, Li K, Yang M and Zhou J: Establishment of clonal colony-forming assay for propagation of pancreatic cancer cells with stem cell properties. Pancreas 34: 429-435, 2007.

25. Rappa G, Mercapide J, Anzanello F, Prasmickaite L, Xi Y, Ju J, Fodstad O and Lorico A: Growth of cancer cell lines under stem cell-like conditions has the potential to unveil therapeutic targets. Exp Cell Res 314: 2110-2122, 2008.

26. Wobser H, Dorn C, Weiss TS, Amann T, Bollheimer C, Büttner R, Schölmerich J and Hellerbrand C: Lipid accumulation in hepatocytes induces fibrogenic activation of hepatic stellate cells. Cell Res 19: 996-1005, 2009.

27. Chong LW, Chou RH, Liao CC, Lee TF, Lin Y, Yang KC and Hsu YC: Saturated fatty acid induces cancer stem cell-like properties in human hepatoma cells. Cell Mol Biol (Noisy-le-Grand) 61: 85-91, 2015.

28. Anderson AM and Ragan MA: Palmitoylation: A protein S-acylation with implications for breast cancer. NPJ Breast Cancer 2: 16028, 2016.

29. Yang MH, Liao CC, Hung JH, Lai XT, Yen CH and Chen YA: Utilizing proteomic approach to identify nuclear translocation related serine kinase phosphorylation site of GNMT as downstream effector for benzo[a]pyrene. J Food Drug Anal 27: 603-609, 2019. 
30. Chong LW, Hsu YC, Lee TF, Lin Y, Chiu YT, Yang KC, Wu JC and Huang YT: Fluvastatin attenuates hepatic steatosis-induced fibrogenesis in rats through inhibiting paracrine effect of hepatocyte on hepatic stellate cells. BMC Gastroenterol 15: 22, 2015.

31. Alessio N, Del Gaudio S, Capasso S, Di Bernardo G, Cappabianca S, Cipollaro M, Peluso G and Galderisi U: Low dose radiation induced senescence of human mesenchymal stromal cells and impaired the autophagy process. Oncotarget 6: 8155-8166, 2015.

32. Buckley BJ and Whorton AR: Tunicamycin increases intracellular calcium levels in bovine aortic endothelial cells. Am J Physiol 273: C1298-C1305, 1997.
33. Sobocińska J, Roszczenko-Jasińska P, Zareba-Kozioł M, Hromada-Judycka A, Matveichuk OV, Traczyk G, Łukasiuk K and Kwiatkowska K: Lipopolysaccharide upregulates palmitoylated enzymes of the phosphatidylinositol cycle: An insight from proteomic studies. Mol Cell Proteomics 17: 233-254, 2018.

34. Patterson SI and Skene JH: Novel inhibitory action of tunicamycin homologues suggests a role for dynamic protein fatty acylation in growth cone-mediated neurite extension. J Cell Biol 124: 521-536, 1994.

This work is licensed under a Creative Commons Attribution-NonCommercial-NoDerivatives 4.0 International (CC BY-NC-ND 4.0) License. 